Carbon monoxide dissociative attachment and resonant dissociation by electron-impact

This content has been downloaded from IOPscience. Please scroll down to see the full text.

2016 Plasma Sources Sci. Technol. 25 01LT04

(http://iopscience.iop.org/0963-0252/25/1/01LT04)

View the table of contents for this issue, or go to the journal homepage for more

Download details:

IP Address: 150.145.83.214

This content was downloaded on 13/01/2016 at $11: 34$

Please note that terms and conditions apply. 


\title{
Letter
}

\section{Carbon monoxide dissociative attachment and resonant dissociation by electron-impact}

\author{
V Laporta ${ }^{1,2,3}$, J Tennyson $^{3}$ and R Celiberto ${ }^{4,2}$ \\ ${ }^{1}$ Ohio Aerospace Institute, Dayton, OH 44142, USA \\ 2 Istituto di Nanotecnologia, CNR, Bari, Italy \\ 3 Department of Physics and Astronomy, University College London, London WC1E 6BT, UK \\ ${ }^{4}$ Dipartimento di Ingegneria Civile, Ambientale, del Territorio, Edile e di Chimica, Politecnico di Bari, \\ 70125 Bari, Italy \\ E-mail: vincenzo.laporta@nanotec.cnr.it
}

Received 21 September 2015, revised 30 November 2015

Accepted for publication 11 December 2015

Published 13 January 2016

\begin{abstract}
Low-energy dissociative electron attachment and resonant electron impact dissociation of $\mathrm{CO}$ molecule are considered. Ro-vibrationally resolved cross sections and rate coefficients for both the processes are calculated using an ab-initio model based on the low-lying $\mathrm{X}^{2} \Pi$ resonance of $\mathrm{CO}^{-}$. Final results show that the cross sections increases very rapidly as a function of the ro-vibrational level; these cross sections should be useful for understanding kinetic dissociation of $\mathrm{CO}$ in strongly non-equilibrium plasmas.
\end{abstract}

Keywords: carbon monoxide, electron scattering, plasma modeling, dissociation, dissociative electron attachment

(Some figures may appear in colour only in the online journal)

Carbon monoxide is a very important molecule playing a fundamental role in many fields. It is, in fact, one of the most abundant species in interstellar medium and can act as a tracer for $\mathrm{H}_{2}$ molecules, which, due to the lack of ground electronic state transition dipole moment, cannot be observed directly but is detected thorough collisions with CO molecules [1]. $\mathrm{CO}$ is present in the atmosphere of planets and comets. It is a component of Mars' and Venus' atmosphere and, in the latter case, represents the most abundant chemical species. It therefore plays a crucial role also in space missions in connection to the (re-)entry problems [2]. Carbon monoxide, finally, is also useful in the understanding of processes involved in $\mathrm{CO}$ laser [3].

In a recent article we published state-resolved cross sections for electron-impact vibrational excitation of carbon monoxide [4]. Those vibrational data are useful in general in plasma vibrational kinetics, where non-thermal conditions can overpopulate the high vibrational levels of $\mathrm{CO}$ molecule [5]. In particular, they have been recently used in modeling the cooling of electrons in non-equilibrium $\mathrm{CO}$-containing flows (see e.g. [6-8]). Since dissociation is an important process in plasma kinetics, as it leads to the depopulation of the vibrational levels, in the present letter we wish to extend our previous work to dissociative electron attachment (DEA) and to resonant electron impact-dissociation (EID) of $\mathrm{CO}$, taking place through the temporary formation of the resonance $\mathrm{CO}^{-}\left(\mathrm{X}^{2} \Pi\right)$. DEA is known to be a relevant process that produces stable negative ions of oxygen [9].

The reactions considered here, respectively for DEA and EID processes, are the following:

$$
\begin{aligned}
& e+\mathrm{CO}\left(\mathrm{X}^{1} \Sigma^{+} ; v, j\right) \rightarrow \mathrm{CO}^{-}\left(\mathrm{X}^{2} \Pi\right) \rightarrow \mathrm{C}\left({ }^{3} \mathrm{P}\right)+\mathrm{O}^{-}\left({ }^{2} \mathrm{P}\right), \\
& e+\mathrm{CO}\left(\mathrm{X}^{1} \Sigma^{+} ; v, j\right) \rightarrow \mathrm{CO}^{-}\left(\mathrm{X}^{2} \Pi\right) \rightarrow e+\mathrm{C}\left({ }^{3} \mathrm{P}\right)+\mathrm{O}\left({ }^{3} \mathrm{P}\right),
\end{aligned}
$$



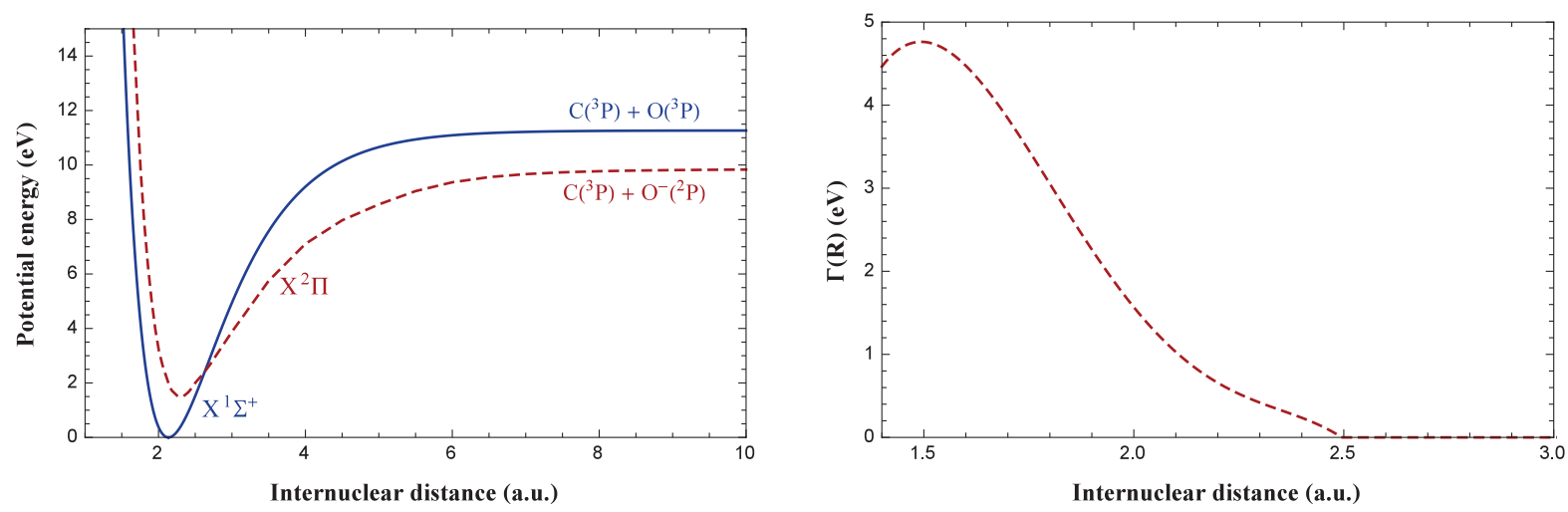

Figure 1. Potential energy curves for the electronic ground state of $\mathrm{CO}$ and for the resonant state $\mathrm{CO}^{-}$(left hand side) and the resonance width (right hand side) taken from [4].

where $v, j$ represent the quantum numbers for the ro-vibrationl levels of the ground electronic state of $\mathrm{CO}$. The vibrationally excited CO molecules, in the DEA process, undergo dissociation with production of a negative oxygen ion while, in the EID process, they decay by emitting an electron which causes vibrational excitation ending in the vibrational continuum of the ground electronic state, then followed by molecular dissociation. The potential energy curves for the neutral and resonant species, $\mathrm{CO}$ and $\mathrm{CO}^{-}$, respectively and the resonance width, $\Gamma(R)$, were obtained in [4] using MOLPRO [10] and the UK Molecular R-Matrix [11] ab-initio quantum chemistry codes. The results are displayed in figure 1 . We calculated also the vibrational energy levels for the neutral molecule, up to dissociation, whose value are given in table 1 for $j=0$. Actually, in addition to the reactions displayed in equations (1) and (2), there is a third dissociative channel which leads to the formation of $\mathrm{C}^{-}+\mathrm{O}$ in the final state. In the following, we neglect this state as the corresponding cross sections are several orders of magnitude lower than the DEA and EID as demonstrated by experimental measurements [12].

The cross sections for the reactions (1) and (2) for electron energy $\epsilon$ are given, respectively, by:

$$
\begin{aligned}
\sigma_{v, j}^{\mathrm{DEA}}(\epsilon) & =2 \pi^{2} \frac{m_{\mathrm{e}}}{k} \frac{K}{\mu} \lim _{R \rightarrow \infty}|\xi(R)|^{2}, \\
\sigma_{v, j}^{\mathrm{EID}}(\epsilon) & =\frac{64 \pi^{5} m_{\mathrm{e}}^{2}}{\hbar^{4}} \int \mathrm{d} \epsilon^{\prime} \frac{k^{\prime}}{k}\left|\left\langle\chi_{\epsilon^{\prime}}(R)|\mathcal{V}| \xi(R)\right\rangle\right|^{2},
\end{aligned}
$$

where: $K$ is the asymptotic momentum of the dissociating fragments $\mathrm{C}$ and $\mathrm{O}^{-}$with reduced mass $\mu ; m_{\mathrm{e}}, k=\sqrt{2 m_{\mathrm{e}} \epsilon / \hbar}$ and $k^{\prime}=\sqrt{2 m_{\mathrm{e}} \epsilon^{\prime} / \hbar}$ are the mass, the incoming and outgoing momentum of the electron; $\xi(R)$ is the solution of the Schrodinger-like equation for the resonant state and total energy $E=\epsilon_{v, j}+\epsilon$ :

$$
\left(-\frac{\hbar^{2}}{2 \mu} \frac{\mathrm{d}^{2}}{\mathrm{~d} R^{2}}+\frac{j(j+1) \hbar^{2}}{2 \mu R^{2}}+V^{-}+\frac{i}{2} \Gamma-E\right) \xi(R)=-\mathcal{V} \chi_{v, j}(R),
$$

where $V^{-}+\frac{i}{2} \Gamma$ is the complex potential of the resonance, whose components are shown in figure $1 ; \mathcal{V}=[\Gamma /(2 \pi k)]^{1 / 2}$ is the discrete-to-continuum potential coupling and $\chi_{v, j}$ is the
Table 1. $\mathrm{CO}$ vibrational levels and the corresponding energies (relative to the state $v=0$ ) for ground electronic state and for $j=0$.

\begin{tabular}{llllllll}
\hline$v$ & $\epsilon_{v, 0}(\mathrm{eV})$ & $v$ & $\epsilon_{v, 0}(\mathrm{eV})$ & $v$ & $\epsilon_{v, 0}(\mathrm{eV})$ & $v$ & $\epsilon_{v, 0}(\mathrm{eV})$ \\
\hline 0 & 0.0000 & 20 & 4.8243 & 40 & 8.2869 & 60 & 10.3877 \\
1 & 0.2735 & 21 & 5.0297 & 41 & 8.4243 & 61 & 10.4570 \\
2 & 0.5437 & 22 & 5.2318 & 42 & 8.5582 & 62 & 10.5229 \\
3 & 0.8104 & 23 & 5.4305 & 43 & 8.6888 & 63 & 10.5854 \\
4 & 1.0738 & 24 & 5.6257 & 44 & 8.8160 & 64 & 10.6445 \\
5 & 1.3337 & 25 & 5.8176 & 45 & 8.9397 & 65 & 10.7002 \\
6 & 1.5902 & 26 & 6.0060 & 46 & 9.0601 & 66 & 10.7524 \\
7 & 1.8434 & 27 & 6.1911 & 47 & 9.1771 & 67 & 10.8013 \\
8 & 2.0931 & 28 & 6.3727 & 48 & 9.2906 & 68 & 10.8468 \\
9 & 2.3394 & 29 & 6.5510 & 49 & 9.4008 & 69 & 10.8888 \\
10 & 2.5823 & 30 & 6.7258 & 50 & 9.5075 & 70 & 10.9275 \\
11 & 2.8218 & 31 & 6.8972 & 51 & 9.6109 & 71 & 10.9628 \\
12 & 3.0580 & 32 & 7.0652 & 52 & 9.7108 & 72 & 10.9946 \\
13 & 3.2907 & 33 & 7.2299 & 53 & 9.8073 & 73 & 11.0231 \\
14 & 3.5200 & 34 & 7.3911 & 54 & 9.9005 & 74 & 11.0481 \\
15 & 3.7459 & 35 & 7.5489 & 55 & 9.9902 & 75 & 11.0697 \\
16 & 3.9684 & 36 & 7.7033 & 56 & 10.0765 & 76 & 11.0880 \\
17 & 4.1874 & 37 & 7.8543 & 57 & 10.1594 & 77 & 11.1028 \\
18 & 4.4031 & 38 & 8.0019 & 58 & 10.2389 & 78 & 11.1142 \\
19 & 4.6154 & 39 & 8.1461 & 59 & 10.3150 & 79 & 11.1222 \\
& & & & & & 80 & 11.1267 \\
\hline
\end{tabular}

Note: The dissociation energies are $D_{\mathrm{e}}=11.266 \mathrm{eV}$ and $D_{0}=11.128 \mathrm{eV}$.

wave function of $\mathrm{CO}$ corresponding to the ro-vibrational level $v, j . R$ represents the internuclear distance. In equation (4), $\langle\cdots\rangle$ means integration over the internuclear distance $R$ and $\chi_{\epsilon^{\prime}}$ is the continuum wave function of $\mathrm{CO}$ with energy $\epsilon^{\prime}$ representing the $\mathrm{C}+\mathrm{O}+e^{-}$fragments. The integration on continuum energy $\epsilon^{\prime}$ was carried out from $\mathrm{CO}$ dissociation threshold, $D_{\mathrm{e}}$, up to $10 \mathrm{eV}$. A similar model was recently used to successfully study DEA and EID in $\mathrm{O}_{2}[13,14]$ and EID in $\mathrm{N}_{2}$ [15].

Figures 2 and 3 summarize the results for $j=0$. In particular, figure 2 (left panel) shows the DEA cross sections for some initial vibrational levels as a function of the incident electron energy. It is evident from the figures that the cross sections for $v=0$ are very small but they increase rapidly for higher vibrational levels. The oscillatory structure is due to the overlap of the vibrational wave functions of the neutral and resonant state [16]. A similar situation is observed for the EID cross sections. Figure 3 (left panel) shows that the variation of 

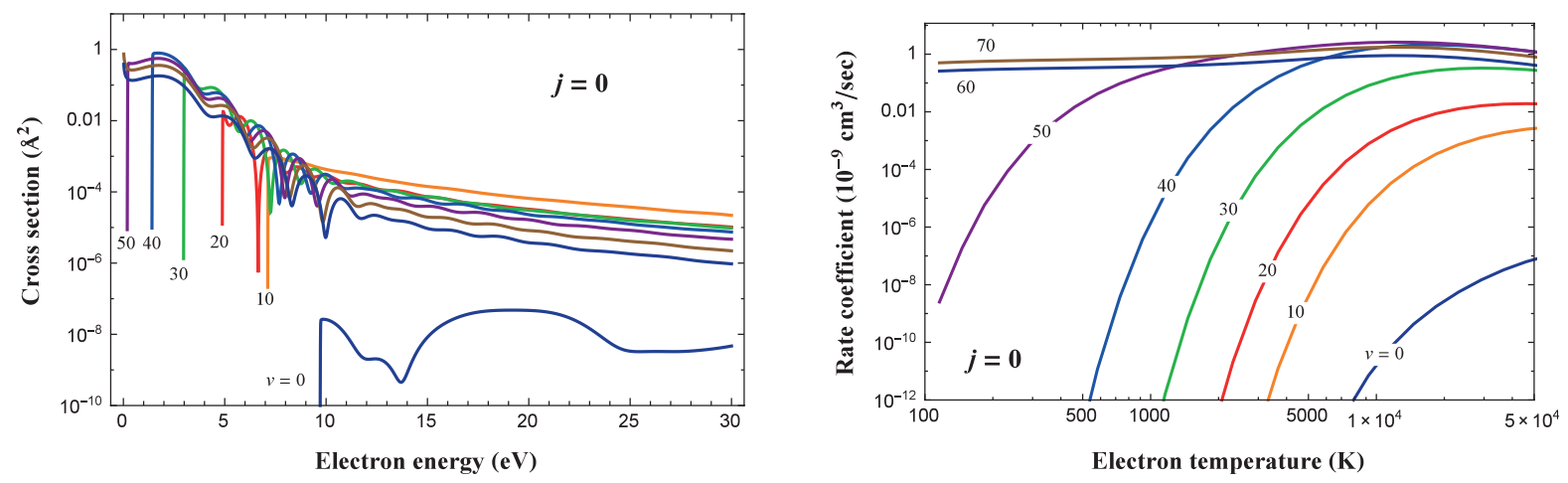

Figure 2. Vibrational state-resolved dissociative electron attachment cross sections and the corresponding rate constants for $j=0$.
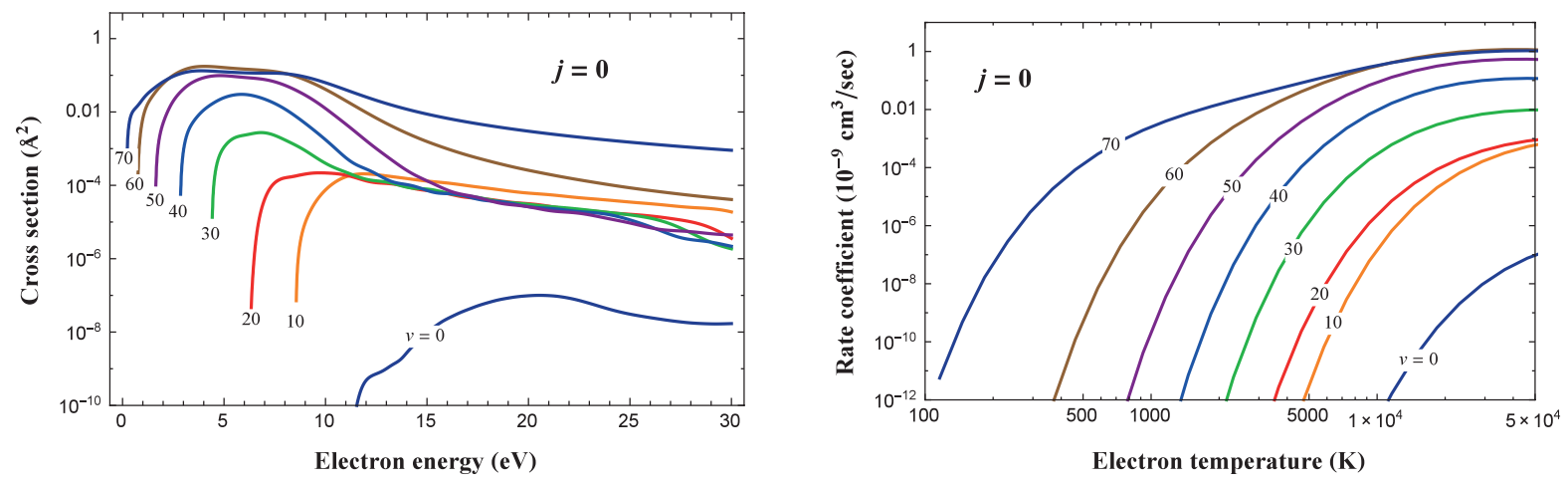

Figure 3. Vibrational state-resolved electron-impact dissociation cross sections and the corresponding rate constants for $j=0$.
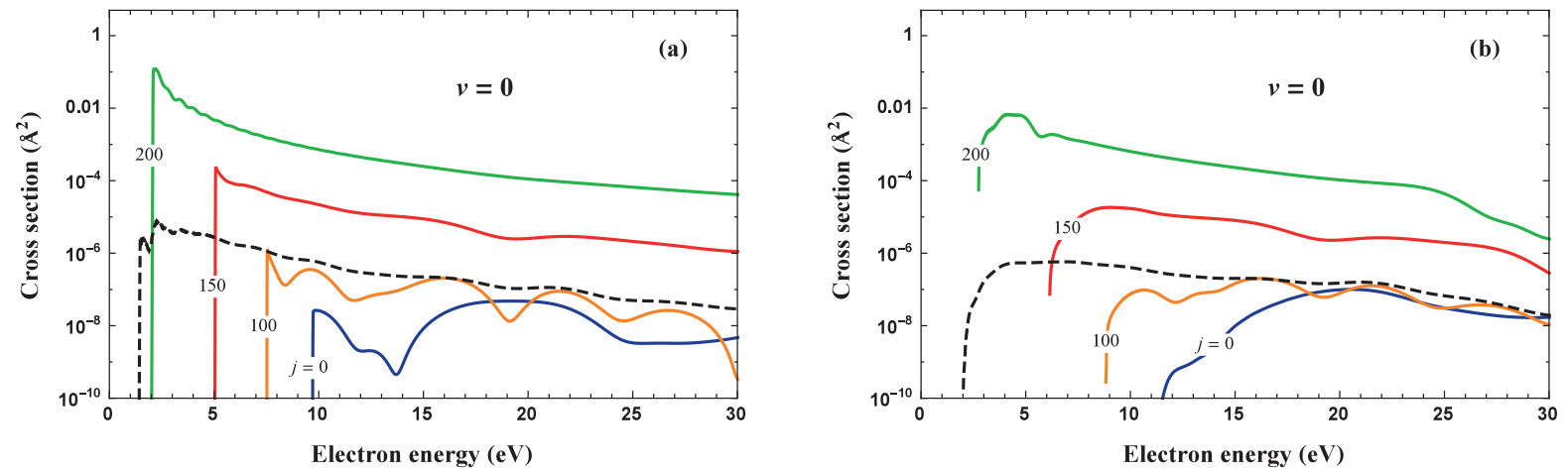

Figure 4. Rotational state-resolved cross sections for $v=0$ : (a) dissociative electron attachment and (b) electron impact dissociation. Dashed black curves refer to the $j$-averaged cross sections calculated by assuming a rotational temperature of $10000 \mathrm{~K}$.

the cross sections with the vibrational level of the molecule is even stronger than for DEA. The different threshold between the corresponding cross sections at fixed $v$ of DEA and EID is due to the electron affinity of oxygen, $E_{a}=1.46 \mathrm{eV}$.

Both the right panels of figures 2 and 3 show, as a function of the electron temperature $T_{\mathrm{e}}$, the rate coefficients for the processes (1) and (2) calculated from the corresponding cross sections assuming a Maxwellian energy distribution for the plasma electrons, i.e.:

$$
\mathrm{k}_{v, j}\left(T_{\mathrm{e}}\right)=\frac{2}{\sqrt{\pi}}\left(\kappa T_{\mathrm{e}}\right)^{-1.5} \int_{\epsilon_{\mathrm{th}}}^{\infty} \epsilon \sigma_{v, j}(\epsilon) \mathrm{e}^{-\epsilon / \kappa T_{\mathrm{e}}} \mathrm{d} \epsilon,
$$

where $\kappa$ is the Boltzmann constant and $\epsilon_{\text {th }}$ is the threshold energy of the process. The absolute value for rate coefficients grow monotonically as the vibrational level $v$ increase and they become very large, particularly for $T_{\mathrm{e}}>1000 \mathrm{~K}$. These rates are important for models of plasmas in strong non-equilibrium. No experimental data are available for resonant dissociation while DEA has been measured by Rapp and Briglia [17] and recently reviewed by Itikawa [18] as part of his compilation of electron-CO cross sections. However the experimental data was obtained at room temperature using a thermal target which means that the small contribution to the process occurring from the $\mathrm{X}^{2} \Pi$ resonance is obscured by the contribution coming from the $\mathrm{A}^{2} \Sigma$ resonant state. DEA from the $\mathrm{A}^{2} \Sigma$ resonance will be the subject of future work.

Cross sections for $j=0$, basically, are valid at room temperature where rotational excitation is low. As the temperature increases rotational effects becomes more important. Figure 4 shows the $j$-dependence of the $v=0$ cross sections for both 
the DEA and EID processes. For low values of $j$, similar oscillatory behaviour to the case of vibration excitations is found. As the rotational quantum number increases, the potential energy curves, including the centrifugal contribution, become less deep and the corresponding cross sections increase in value while the threshold of the dissociative process is reduced. Dashed curves in figure 4 refer to the $j$-averaged cross sections obtained by assuming a rotational temperature $T_{\mathrm{R}}=10000 \mathrm{~K}$ and $v=0$ :

$$
\bar{\sigma}_{v}\left(T_{\mathrm{R}}, \epsilon\right)=\sum_{j} \sigma_{v, j}(\epsilon)(2 j+1) \mathrm{e}^{-\epsilon_{v, j} / \kappa T_{\mathrm{R}}} .
$$

In conclusion, in the present letter we have extended our previous work in [4] on vibrational excitation of CO molecule by electron-impact, by calculating the cross sections and the corresponding rates constants for the dissociative electron attachment and electron impact-dissociation processes. Understanding these processes is very important in modeling non-equilibrium plasma as they represent the principal reactions that lead to direct break of $\mathrm{CO}$ molecule antagonist to the so-called 'pure-vibrational-mechanism' for dissociation [7]. The full set of data obtained in the present work is available via the website of the Phys4Entry project [19] and as supplementary material to this letter.

\section{Acknowledgments}

The authors wish to thank Prof M Capitelli (University of Bari, Italy) for useful discussions. Research of VL was funded by Ohio Aerospace Institute, grant agreement project \#WE202270.

\section{References}

[1] Burgh E B, France K and McCandliss S R 2007 Astrophys. J. $\mathbf{6 5 8} 446$

[2] Campbell L, Allan M and Brunger M J 2011 J. Geophys. Res. 116 A09321

[3] Haddad G N and Milloy H B 1983 Aust. J. Phys. 36473

[4] Laporta V, Cassidy C M, Tennyson J and Celiberto R 2012 Plasma Sources Sci. Tech. 21045005

[5] Essenhigh K A, Utkin Y G, Bernard C, Adamovich I V and Rich J W 2006 Chem. Phys. 330 506-14

[6] Graetz F, Engelhart D P, Wagner R J V, Meijer G, Wodtke A M and Schafer T 2014 J. Chem. Phys. 141044712

[7] Capitelli M, Colonna G, D'Ammando G, Laporta V and Laricchiuta A 2014 Chem. Phys. 438 31-6

[8] Kozak T and Bogaerts A 2015 Plasma Sources Sci. Tech. 24015024

[9] Aleksandrov N L and Anokhin E M 2009 J. Phys. D: Appl. Phys. 42225210

[10] Werner H-J et al 2010 MOLPRO, version 2010.1, a package of ab initio programs

[11] Tennyson J 2010 Phys. Rep. 491 29-76

[12] Stamatovic A and Schulz G J 1970 J. Chem. Phys. 53 2663-7

[13] Laporta V, Celiberto R and Tennyson J 2015 Phys. Rev. A 91012701

[14] Laporta V, Celiberto R and Tennyson J 2014 AIP Conf. Proc. 1628 939-42

[15] Laporta V, Little D A, Celiberto R and Tennyson J 2014 Plasma Sources Sci. Tech. 23065002

[16] Celiberto R, Janev R K, Laporta V, Tennyson J and Wadehra J M 2013 Phys. Rev. A 88062701

[17] Rapp D and Briglia D D 1965 J. Chem. Phys. 43 1480-9

[18] Itikawa Y 2015 J. Phys. Chem. Ref. Data 44013105

[19] Database of the european union phys4entry project 2012-2015 http://users.ba.cnr.it/imip/cscpal38/phys4entry/database. html 